\title{
Glutathione improves the function of porcine pulmonary grafts stored for twenty-four hours in low-potassium dextran solution
}

\author{
Sebastian-P. Sommer, MD, ${ }^{a}$ Bernhard Gohrbandt, MD, ${ }^{a}$ Stefan Fischer, MD, MSc, ${ }^{a}$ Jens M. Hohlfeld, MD,
} Gregor Warnecke, MD, ${ }^{a}$ Murat Avsar, MD, ${ }^{a}$ and Martin Strüber, $\mathrm{MD}^{\mathrm{a}}$

From the Hannover Thoracic Transplant Program, Division of Thoracic and Cardiovascular Surgery, ${ }^{\mathrm{a}}$ and Department of Respiratory Medicine, ${ }^{\mathrm{b}}$ Hannover Medical School, Hannover, Germany.

Received for publication Feb 12, 2005; revision received May 11, 2005; accepted for publication May 16, 2005.

Address for reprints: Martin Strüber, MD, Director, Hannover Thoracic Transplant Program, Division of Thoracic and Cardiovascular Surgery, Hannover Medical School, 30623 Hannover, Germany (E-mail strueber@thg.mh-hannover.de).

J Thorac Cardiovasc Surg 2005;130:864-9

$0022-5223 / \$ 30.00$

Copyright (C) 2005 by The American Association for Thoracic Surgery

doi:10.1016/j.jtcvs.2005.05.021
Background: Flush perfusion with low-potassium dextran is the standard strategy in clinical lung preservation. Despite improved outcome, endothelial cell injury and surfactant dysfunction remain a significant problem after lung transplantation. The radical scavenger glutathione has been shown to be responsible for the efficacy of Celsior solution in lung preservation. We tested the hypothesis that the addition of glutathione to low-potassium dextran might further improve graft function by ameliorating ischemia-reperfusion injury.

Methods: In 12 domestic pigs, lungs were flush preserved with either low-potassium dextran $(\mathrm{n}=6)$ or low-potassium dextran supplemented by 5 mmol glutathione $(\mathrm{n}$ $=6$ ). Left single lung transplantation was performed after 24-hour storage in low-potassium dextran at $8^{\circ} \mathrm{C}$. After 15 minutes of reperfusion the right main bronchus and pulmonary artery were crossclamped. Hemodynamic and respiratory measures were recorded in 30-minute intervals for a total observation period of 7 hours. Bronchoalveolar lavage fluid was obtained from the native lung and 2 hours after reperfusion from the graft. Bronchoalveolar lavage fluid and surfactant composition, and surfactant function analyses were performed. Neutrophil sequestration was assessed by myeloperoxidase activity assay. Tissue water content was calculated from wet/dry weight ratios at the end of the experiment.

Results: In the low-potassium dextran group, 2 animals died during reperfusion. After reperfusion, pulmonary vascular resistance $(P=.01)$ and pulmonary artery pressure remained lower in the glutathione/low-potassium dextran group, which was associated with a higher cardiac output $(P=.05)$ in this group. Also, the oxygenation index at the end of the observation period was higher in the glutathione/lowpotassium dextran group compared with the low-potassium dextran group (430 \pm 130 vs $338 \pm 184$, respectively; $P<.05$ ). The graft water content representing postreperfusion lung edema was not different between the 2 study groups. Alteration of surfactant was less in the glutathione/low-potassium dextran group with a significantly decreased small to large aggregate ratio $(P=.03)$ versus low-potassium dextran group. Myeloperoxidase activity was twice as high in the low-potassium dextran group when compared with the glutathione/low-potassium dextran group (glutathione/low-potassium dextran: $134 \pm 110 \mathrm{mU} / \mathrm{g}$ vs low-potassium dextran: $274 \pm 168 \mathrm{mU} / \mathrm{g}, P=.07)$.

Conclusion: The addition of glutathione to low-potassium dextran preservation solution reveals beneficial effects on vascular function and surfactant composition in transplanted lungs. Therefore, glutathione ameliorates ischemia-reperfusion injury in a preclinical model of lung transplantation. Future studies are needed to evaluate this promising modification in clinical lung transplantation. 
I schemia-reperfusion (I/R) injury remains a serious issue in pulmonary transplantation. ${ }^{1,2}$ The use of lowpotassium dextran (LPD) solution for pulmonary preservation has become a standard strategy in clinical lung transplantation leading to improved outcome, as reported by us and others. ${ }^{3-5}$ Recently, we demonstrated that the use of Celsior solution leads to superior pulmonary-vascular preservation when compared with LPD in experimental lung transplantation. ${ }^{6}$ Because I/R injury is mediated in part by the generation of oxygen-derived radicals, oxidizing enzymes, or nitric oxide (NO), ${ }^{7}$ we addressed the beneficial effect of Celsior preservation solution on pulmonaryvascular function to the antioxidative capability of its additive, reduced glutathione (GSH). Because of the characteristic inflammatory response to $\mathrm{I} / \mathrm{R}$ in transplanted lungs, depletion of reductive thiols (ie, GSH) may occur. ${ }^{8}$ Supplementation of GSH has an inhibitory effect on oxygenderived radicals. This, consequently, preserves cellular function and cell integrity. In addition, GSH enhances endothelium-dependent NO release by improving the transmembrane transportation of NO into smooth muscle cells. ${ }^{8}$ However, in comparison with Celsior solution, LPD seems to provide superior protection of the surfactant system because of its component dextran 40. As previously reported, dextran 40 acts as an impermeant preventing extravasation of water into the alveolar space, thereby preventing surfactant inhibition. ${ }^{9}$ In addition, it protects the graft from intravascular microthrombi formation by coating corpuscular blood components. ${ }^{9}$ We hypothesized that the addition of GSH to LPD solution further improves pulmonary preservation in a preclinical model of single lung transplantation compared with LPD alone.

\section{Methods}

The purpose of this study was to evaluate initial lung function after 24-hour cold ischemic preservation with LPD or GSH-enriched LPD solution followed by a 7-hour observational reperfusion period. In addition, surfactant function analysis was performed, which has repeatedly been shown to be a sensitive parameter for the quality of lung preservation.

\section{Animal Care}

All animals received humane care in compliance with the "Principles of Laboratory Animal Care" and the "Guide for the Care and Use of Laboratory Animals" prepared by the Institute of Laboratory Animal Resources, National Research Council, and published by the National Academy Press, revised 1996. The study protocol was reviewed, and all experiments were approved by the local ethics committee of the Hannover Medical School.

\section{Donor Procedure}

In 12 female pigs (German Landrace; $24-33 \mathrm{~kg}$ ), anesthesia was induced by the application of azaperone $(5 \mathrm{mg} / \mathrm{kg}$, intramuscularly), atropine (5 mg total dose, intramuscularly), and pentobarbital (1 mg/kg, intravenously). Animals were intubated and venti- lated in a pressure-controlled mode with a peak inspiratory pressure of $30 \mathrm{cmH}_{2} \mathrm{O}$, a positive end-expiratory pressure of 5 $\mathrm{cmH}_{2} \mathrm{O}$, and an $\mathrm{FIO}_{2}$ of 0.5 . Anesthesia was maintained by continuous infusion of pentobarbital $(5 \mathrm{mg} \cdot \mathrm{kg} \cdot \mathrm{h})$ and fentanyl $(1$ $\mu \mathrm{g} \cdot \mathrm{kg} \cdot \mathrm{h}$ ). After median sternotomy, the inferior and superior venae cavae were encircled with ties and the pulmonary artery was dissected from the ascending aorta. After systemic application of heparin $(300 \mathrm{IU} / \mathrm{kg}$ ), a 5-mm cannula was inserted into the pulmonary artery. Right heart inflow occlusion was performed, and the left atrial appendage was excised. The pulmonary artery was clamped and either $1 \mathrm{~L}$ of $4^{\circ} \mathrm{C}$ cold LPD (Perfadex, Vitrolife, Gothenburg, Sweden) supplemented by $0.3 \mathrm{~mL}$ Tris-buffer or $1 \mathrm{~L}$ of $4{ }^{\circ} \mathrm{C}$ cold GSH-LPD (Sigma-Aldrich Laborchemikalien GmbH, Germany) with 0.7 to $1.1 \mathrm{~mL}$ Tris-buffer was infused. The $\mathrm{pH}$ of the preservation solution was adjusted using Tris-buffer to a target level of 7.5. The intrapulmonary artery pressure was recorded throughout the flushing period. A mean perfusion pressure of 16 $\mathrm{mm} \mathrm{Hg}$ was maintained. The harvested lungs were stored in a semi-inflated state in LPD at $4^{\circ} \mathrm{C}$ for 24 hours.

\section{Porcine Single Lung Transplantation}

In 12 female pigs (German Landrace; $23-32 \mathrm{~kg}$ ), anesthesia was induced and maintained as described. Recipient arterial pressure was monitored by a carotid artery catheter, and pulmonary artery hemodynamics were monitored by a Swan-Ganz catheter $(7.5 \mathrm{~F}$, Baxter Healthcare, Irvine, Calif). Cardiac output and extravasal lung water were recorded with a femoral artery thermodilution catheter connected to a cardiac output recording device (Picco System; Pulsion Medical Systems AG, Munich, Germany). The chest was entered through a left lateral thoracotomy in the fourth intercostal space. Left pulmonary artery, lung veins, and bronchus were dissected. After systemic administration of heparin (300 IU/kg), a left-sided pneumonectomy was performed. The graft was transplanted with running polypropylene sutures used for all 3 anastomoses. After 10 minutes of reperfusion the right pulmonary artery and right main bronchus were crossclamped.

\section{Assessment of Hemodynamics and Lung Function}

Right atrial and arterial and pulmonary artery pressures were recorded online. Arterial and venous blood gas analyses were performed in 30-minute intervals after reperfusion. Pulmonary vascular resistance (PVR) was calculated. The system was calibrated by 3 repeated bolus injections of $10 \mathrm{~mL}$ of $8^{\circ} \mathrm{C}$ cold saline solution into the jugular vein. Experiments were terminated by a pentobarbital overdose after 7 hours of reperfusion.

\section{Surfactant, Protein, and Phospholipid Analysis}

A bronchoalveolar lavage in the native recipient lingula was performed with $100 \mathrm{~mL}$ warmed isotonic saline solution before transplantation. A second bronchoalveolar lavage of the lingula of the transplanted lung was performed 2 hours after reperfusion. The recovered bronchoalveolar lavage fluid (BALF) was centrifuged at $150 \mathrm{~g}$, and the cell-free supernatant was frozen at $-80^{\circ} \mathrm{C}$ until further analysis. From the cell pellet a manual differential cell count was performed by standard techniques. From the cell-free supernatant samples, phospholipid content was determined according to the method described by Bartlett. ${ }^{10}$ Protein content was measured according to the technique described by Lowry and 


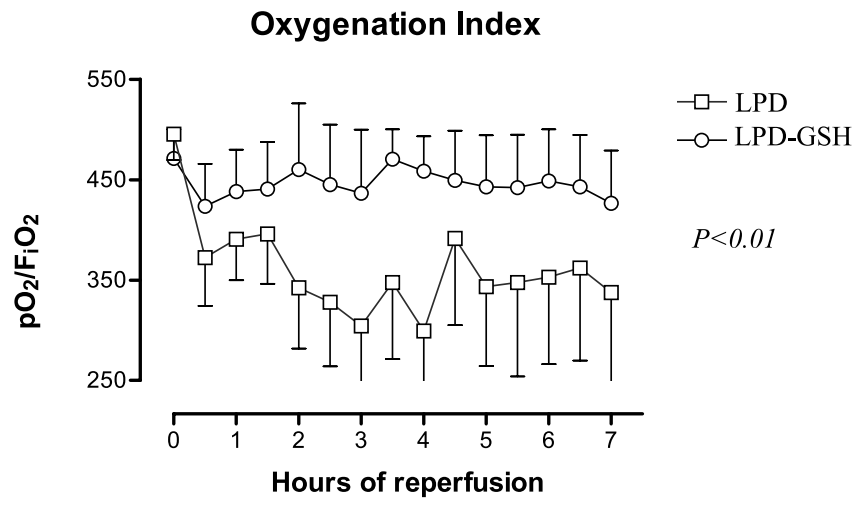

Figure 1. $\mathrm{Po}_{2} / \mathrm{FiO}_{2}$ ratio in arterial blood samples drawn from the left atrium over the 7-hour reperfusion period. LPD, Low-potassium dextran; $\mathbf{G S H}$, glutathione.

associates. ${ }^{11}$ All assays were performed as duplicate measures, and the mean value was reported. Surfactant was isolated from BALF by centrifugation at $48,000 \mathrm{~g}$ for 60 minutes at $4^{\circ} \mathrm{C}$. Phospholipid determination of the pellet and the supernatant served for calculation of the small to large aggregate (SA/LA) ratio. Surfactant function was determined by a pulsating bubble surfactometer (Electronetics, Buffalo, NY) according to the technique described by Enhorning. ${ }^{12}$ In brief, $40 \mu \mathrm{L}$ of the pelleted surfactant, which had been adjusted to a phospholipid concentration of $1 \mathrm{mg} / \mathrm{mL}$, was filled into the sample chamber. The surface tension at minimal bubble size ( $\gamma \mathrm{min})$ was recorded after 5 minutes of bubble pulsation at a rate of $20 \mathrm{cycles} / \mathrm{min}$ and a temperature of $37^{\circ} \mathrm{C}$. Before bubble pulsation was started the adsorption rate was determined as surface tension 10 seconds after formation of a bubble ( $\gamma$ ads). All analog data were digitalized and recorded.

\section{Myeloperoxidase Activity Assay}

Relative neutrophil sequestration into lung tissue was assessed by a myeloperoxidase activity assay. ${ }^{13}$ Frozen lung tissue specimen was homogenized in $1.5 \mathrm{~mL}$ of $0.02 \mathrm{~mol} / \mathrm{L}$ potassium phosphate buffer (pH 7.4). The suspension was centrifuged at $10,000 \mathrm{~g}$ for 15 minutes. The supernatant was discarded, and the pellet was resuspended in $2 \mathrm{~mL}$ of $0.5 \%$ hexadecyltrimethylammonium bromide in $50 \mathrm{mmol} / \mathrm{L}$ potassium phosphate solution $(\mathrm{pH} 6.0)$ and homogenized. Tissue was disrupted by sonication and 3 freeze-thaw cycles (liquid nitrogen bath $/ 37^{\circ} \mathrm{C}$ water bath). The suspension was centrifuged at $10,000 \mathrm{~g}$ for 15 minutes. Aliquots $(0.1 \mathrm{~mL})$ of supernatant were added to $1 \mathrm{~mL}$ of tetramethylbenzidine substrate system (Sigma Chemical; St Louis, Mo) at pH 6.0. The change in absorption at $655 \mathrm{~nm}$ at $25^{\circ} \mathrm{C}$ over 3 minutes was recorded. Assays were performed as repeated measures and results are expressed as means in milliunits per gram.

\section{Statistical Analysis}

Data were expressed as mean \pm standard error of the mean. Analysis of continuous data was performed using repeatedmeasures analysis of variance (ANOVA). Data without repeated measurements were analyzed by the 2 -sided Student $t$ test. All data were analyzed with the Scientific Program of Social Sciences (SPSS for Windows version 10.0; SPSS Inc, Chicago, Ill).

\section{Results}

\section{Animal Survival}

In the LPD group, 2 animals died of right heart failure caused by severe reperfusion injury 2.5 and 4 hours after reperfusion, whereas in the GSH-LPD group all 6 animals survived for the entire experiment.

\section{Hemodynamic and Respiratory Parameters}

The arterial oxygenation index remained lower in grafts preserved with LPD compared with GSH-LPD throughout the observation time. At the end of the reperfusion period, LPD-preserved lungs showed a lower $\mathrm{Po}_{2} / \mathrm{FIO}_{2}$ ratio of $338 \pm$ 184 compared with GSH-LPD-preserved grafts (430 \pm 130), as shown in Figure $1(P<.01)$. Dynamic lung compliance was comparable in both experimental groups during the observation period. An early increase in PVR after reperfusion resulted in right heart failure in 2 animals in the LPD group. Whereas the 6 GSH-LPD-treated lungs reached a maximum in PVR of $659 \pm 72 \mathrm{dyn} \cdot \mathrm{s} \cdot \mathrm{cm}^{-5}$, LPD grafts showed significantly higher values with a peak at $1573 \pm 621 \mathrm{dyn} \cdot \mathrm{s} \cdot \mathrm{cm}^{-5}(P=.01)$ after graft reperfusion (Figure 2).

\section{Lung Water Content}

Lung tissue water content was significantly increased in both groups at the end of the experiment compared with control lungs. However, no statistical difference was achieved between the LPD and GSH-LPD groups (wet/dry ratio: LPD: $8.1 \pm 0.8$ vs LPD-GSH: $7.5 \pm 3.3, P=.11$ ).

\section{BALF Analysis}

BALF from native lungs showed normal cell counts with a predominance of alveolar macrophages $(86 \%)$ and approximately $3.6 \%$ neutrophils (Table 1 ). After 2 hours of reperfusion, a reduced percentage of macrophages $(80 \% \pm 8.6 \%$ LPD vs $73.3 \% \pm 26.2 \%$ GSH-LPD, $P=.57$ ) combined with a substantial increase in relative neutrophil count $(26.8 \% \pm 28.0 \%$ LPD vs $21.3 \% \pm 23.1 \%$ GSH-LPD, $P=$ .72) was observed in groups (Table 1). The relative increase of neutrophils was equal in both study groups. However, the total cell count in the GSH-LPD group remained substantially lower after reperfusion when compared with native controls and LPD-preserved lungs, indicating a lower absolute neutrophil count in the GSH-LPD group (Table 1). Differences between both groups and native lungs did not reach statistical significance. The conversion of surface active surfactant aggregates into surface inactive small surfactant aggregates is expressed by the SA/LA ratio. Control lung values revealed a low quotient of $0.2 \pm 0.1$. Surfactant analysis after 2 hours of reperfusion revealed a 2- to 9-fold increase of the SA/LA ratio $(1.8 \pm 1.4 \mathrm{LPD}$ vs $0.4 \pm 0.3$ 


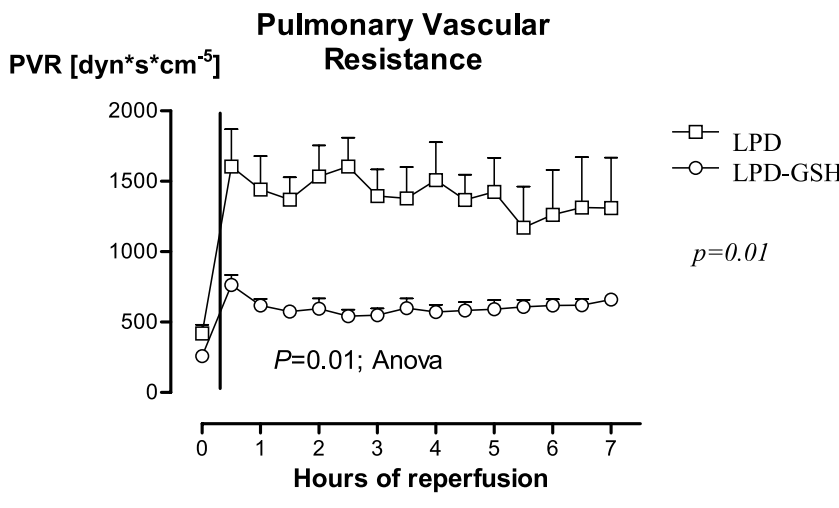

Figure 2. PVR over 7 hours of reperfusion. Clamping the right main pulmonary artery caused a significant increase in PVR in both groups. Differences in PVR over 7 hours were statistically significant between both groups $(P=.01)$. $L P D$, Low-potassium dextran; GSH, glutathione; PVR, pulmonary vascular resistance; Anova, analysis of variance.

GSH-LPD; $P=.04$ ) when compared with control lungs with a trend toward higher levels in the GSH-LPD group $(P$ $=.15$ ) but a substantial amplification (control vs LPD, $P=$ .05 ) in the LPD group (Figure 3).

\section{Surface Activity}

After 5 minutes of pulsation time, the surface tension at minimal bubble size was $1.6 \pm 0.6 \mathrm{mN} / \mathrm{m}$ in control lungs. After 2 hours of reperfusion, surface tension increased to $8.0 \pm 6.7 \mathrm{mN} / \mathrm{m}$ in the LPD group, whereas in the GSHLPD group the increment appeared less pronounced with a value of $5.2 \pm 8.6 \mathrm{mN} / \mathrm{m}$. However, differences between groups were not statistically significant.

\section{Myeloperoxidase Activity}

Myeloperoxidase activity in lung tissue reflects sequestration of neutrophil granulocytes. Tissue samples of control lungs showed a low myeloperoxidase activity $(7.3 \pm 5.6$ $\mathrm{mU} / \mathrm{g}$ ). After 7 hours of reperfusion a substantial increase in myeloperoxidase activity to the 13-fold (GSH-LPD) and 38 -fold (LPD) was seen compared with control lungs $(P<$ .04). Differences between both study groups did not reach

TABLE 1. Differential cell count obtained from BALF 2 hours after reperfusion

\begin{tabular}{lccc}
\hline & LPD & LPD-GSH & Control \\
\hline Macrophages (\%) & $80.0 \pm 8.6$ & $73.3 \pm 26.2$ & $86.0 \pm 11.6$ \\
Neutrophils (\%) & $26.8 \pm 28.0$ & $21.3 \pm 23.1$ & $3.6 \pm 5.0$ \\
Cell count (cells/ $\mu \mathrm{L})$ & $122.4 \pm 94.6$ & $15.5 \pm 5.8$ & $415.0 \pm 280$
\end{tabular}

$\angle P D$, Low-potassium dextran; GSH, glutathione. Data are expressed as mean \pm standard error of mean. Values did not differ significantly.

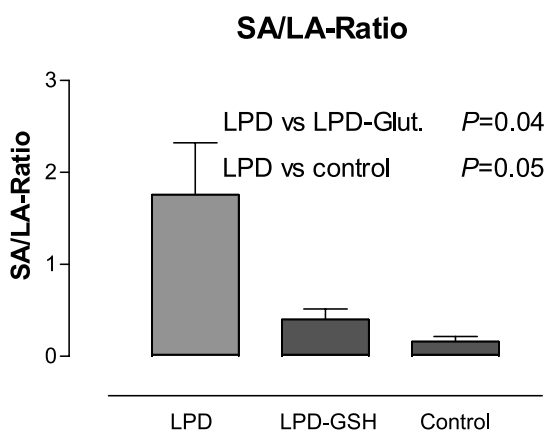

Figure 3. SA/LA ratio of the BALF phospholipid composition 2 hours after reperfusion compared with control BALF collected from recipient native lungs. $S A / L A$, Small to large aggregate; $L P D$, low-potassium dextran; GSH, glutathione.

statistical significance $(274 \pm 168 \mathrm{mU} / \mathrm{g}, \mathrm{LPD}$ vs $94 \pm 89$ $\mathrm{mU} / \mathrm{g}$, GSH-LPD; $P=.06$ ) (Figure 4).

\section{Discussion}

In the pursuit of further improvement in lung preservation, many strategies have been developed and described. ${ }^{14,15}$ In this study, the effect of a GSH as an additive to LPD solution on posttransplant graft function, I/R injury, and surfactant function were compared with LPD only, which is currently the gold standard in clinical lung preservation. ${ }^{4,5}$ We used our previously described preclinical porcine single lung transplant model, which has the obvious advantage of reflecting the clinical situation more precisely than small animal transplant models. ${ }^{16}$ We wanted to inflict a major ischemic injury on the graft, and therefore a 24-hour period of cold ischemic preservation was chosen. The model includes a 7-hour graft reperfusion period, throughout which repeated hemodynamic, laboratory, and histologic measurements and examinations were performed. Therefore, this

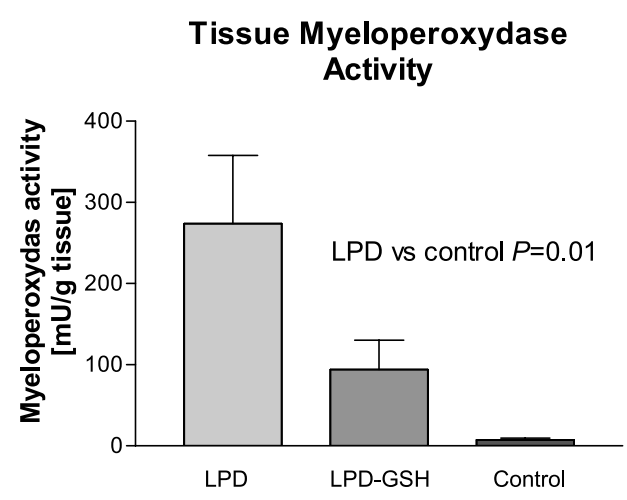

Figure 4. Myeloperoxidase activity of lung tissue after termination of the experiment to control tissue collected from recipient native lungs. $L P D$, Low-potassium dextran; GSH, glutathione. 
preclinical large animal model best mimics $\mathrm{I} / \mathrm{R}$ injury in transplanted lungs, which is a major contributor to early mortality in clinical lung transplantation. ${ }^{17} \mathrm{High}$ potassiumtype solutions such as Euro-Collins have initially been applied for lung graft preservation. We and others, however, have repeatedly reported on the disadvantages of EuroCollins-based lung preservation and have specifically shown a superior effect of LPD on endothelial lung injury and surfactant function in the transplanted lung, which is associated with a corresponding improvement of $\mathrm{I} / \mathrm{R}$ injury. ${ }^{18,19}$ Thus, the introduction of the extracellular type LPD solution significantly improved the outcome in experimental lung preservation with prolonged cold ischemic times up to 24 hours as well as clinical outcome after lung transplantation. ${ }^{4,5,20-22}$

We recently demonstrated that Celsior, a GSH-based preservation solution, provides reliable pulmonary protection. ${ }^{6}$ Thereby, Celsior led to improved protection of pulmonary vascular function, whereas LPD was more protective of the pulmonary surfactant system. We speculated that the improvement in vascular function after Celsior application had to be addressed to its additive, GSH, which is a potent oxygen free radical scavenger. ${ }^{8}$ In this study we examined the effect of reduced GSH as an additive to LPD for pulmonary preservation on posttransplant lung function after extended cold ischemic preservation using a preclinical large animal model. In its action as a radical scavenger, GSH protects the endothelium from oxygen-derived free radical damage. ${ }^{23,24}$ Furthermore, it reduces neutrophil sequestration and acts as a potent vasodilator by improving NO bioavailability and liberation to smooth muscle cells. Compared with LPD-preserved lungs, Celsior-preserved grafts demonstrated improved vascular function by the reduction of both pulmonary artery pressure and PVR. However, surfactant function was better preserved in LPDpreserved lungs. Ideally, GSH-enriched LPD may have the beneficial effect of improving both vascular and surfactant function in transplanted lungs. Indeed, we showed in this study that crucial parameters of posttransplant pulmonary function such as systemic oxygenation and endothelial function were superiorly protected by GSH-LPD compared with LPD-preserved lungs. Furthermore, surfactant function was also better preserved than with LPD alone, as demonstrated by the SA/LA ratio. With regard to the other end points, GSH remarkably improved the protective action of LPD. Whereas in the LPD group 2 of 6 animals died 2.5 hours and 4 hours after reperfusion from right ventricular failure caused by I/R injury-related increase of PVR, the entire GSH-LPD cohort survived for the 7-hour observation period. Parameters of posttransplant lung function, such as the $\mathrm{FIO}_{2} / \mathrm{PAO}_{2}$ ratio, were significantly improved in the $\mathrm{GSH}$ group accompanied by significantly decreased PVR and pulmonary artery pressure, and an increased cardiac output.
At the end of the 7-hour reperfusion period, the pulmonary water content as assessed by wet/dry lung weight ratios was larger in the LPD group. Neutrophil sequestration remained lower to some extent in the LPD-GSH group than the LPD group, respectively. Surfactant activity showed uniform impairment in both groups as shown by an increased minimal surface tension $\left(\gamma_{\min }\right)$ as determined in the pulsating bubble surfactometer. Also, an increase of the SA/LA ratio of the phospholipid fraction was seen. However, overall only mild surfactant dysfunction was observed in both study groups.

In previous studies we extensively evaluated preservation solutions for lung preservation. We and other clinical lung transplant program investigators are currently focusing on the extension of donor criteria to increase the number of transplantable donor organs. Therefore, an increasing number of elderly or "marginal" donors are accepted for transplantation. ${ }^{25}$ This, however, necessitates optimal preservation to exclude the additional confounding factor of preservation injury. Therefore, we strongly believe that the issue of lung preservation is even more important in lung transplantation than ever before. We strive to improve our current standard in lung preservation toward extended ischemic times, which will ultimately help to procure organs throughout Europe. This study is our first attempt to improve our current clinical standard in lung preservation. We are encouraged by our results, and the improvement after lung transplantation with the application of the relatively simple modification of LPD with GSH is remarkable. Ongoing work in our laboratory is now directed toward elucidating the mechanisms involved.

\section{Conclusion}

GSH supplementation of LPD is a reasonable strategy to further improved lung preservation leading to superior graft function and reduced posttransplant I/R injury. The use of GSH-enriched LPD in clinical lung transplantation seems to be a desirable approach especially with regard to prolonged ischemic preservation.

We thank Ms. Petra Oppelt for reviewing statistical analysis as a biostatistician. We furthermore acknowledge that this study would not have been possible without the professional help of the Hannover Experimental Lung Transplant Group.

\section{References}

1. King RC, Binns OA, Rodriguez F, Kanithanon RC, Daniel TM, Spotnitz WD, et al. Reperfusion injury significantly impacts clinical outcome after pulmonary transplantation. Ann Thorac Surg. 2000;69: 1681-5.

2. Struber M, Hohlfeld JM, Kofidis T, Warnecke G, Niedermeyer J, Sommer SP, et al. Surfactant function in lung transplantation after 24 hours of ischemia: advantage of retrograde flush perfusion for preservation. J Thorac Cardiovasc Surg. 2002;123:98-103.

3. Struber M, Hohlfeld JM, Fraund S, Kim P, Warnecke G, Haverich A. Low-potassium dextran solution ameliorates reperfusion injury of the 
lung and protects surfactant function. J Thorac Cardiovasc Surg. 2000;120:566-72.

4. Fischer S, Matte-Martyn A, De Perrot M, Waddell TK, Sekine Y, Hutcheon M, et al. Low-potassium dextran preservation solution improves lung function after human lung transplantation. J Thorac Cardiovasc Surg. 2001;121:594-6.

5. Struber M, Wilhelmi M, Harringer W, Niedermeyer J, Anssar M, Kunsebeck A, et al. Flush perfusion with low potassium dextran solution improves early graft function in clinical lung transplantation. Eur J Cardiothorac Surg. 2001;19:190-4.

6. Sommer SP, Warnecke G, Hohlfeld JM, Gohrbandt B, Niedermeyer J, Kofidis T, et al. Pulmonary preservation with LPD and Celsior solution in porcine lung transplantation after $24 \mathrm{~h}$ of cold ischemia. Eur J Cardiothorac Surg. 2004;26:151-7.

7. Li C, Wright MM, Jackson RM. Reactive species mediated injury of human lung epithelial cells after hypoxia-reoxygenation. Exp Lung Res. 2002;28:373-89.

8. Prasad A, Andrews NP, Padder FA, Husain M, Quyyumi AA. Glutathione reverses endothelial dysfunction and improves nitric oxide bioavailability. J Am Coll Cardiol. 1999;34:507-14.

9. Steen S, Kimblad PO, Sjoberg T, Lindberg L, Ingemansson R, Massa G. Safe lung preservation for twenty-four hours with Perfadex. Ann Thorac Surg. 1994;57:450-7.

10. Bartlett GR. Phosphorous assay in column chromatography. J Biol Chem. 1959;234:466-8.

11. Lowry OH, Rosebrough NJ, Farr AL, Randall RJ. Protein measurement with the Folin phenol reagent. J Biol Chem. 1951;193:265.

12. Enhorning G. Pulsating bubble technique for evaluating pulmonary surfactant. J Appl Physiol. 1977;43:198-203.

13. Kuebler WM, Abels C, Schuerer L, Goetz AE. Measurement of neutrophil content in brain and lung tissue by a modified myeloperoxidase assay. Int J Microcirc Clin Exp. 1996;16:89-97.

14. Date H, Matsumura A, Manchester JK, Obo H, Lima O, Cooper JM, et al. Evaluation of lung metabolism during successful twenty-fourhour canine lung preservation. J Thorac Cardiovasc Surg. 1993;105: 480-91.
15. Jones MT, Hsieh C, Yoshikawa K, Patterson GA, Cooper JD. A new model for assessment of lung preservation. J Thorac Cardiovasc Surg. 1988;96:608-14.

16. Warnecke G, Sommer SP, Gohrbandt B, Fischer S, Hohlfeld JM, Niedermeyer J, et al. Warm or cold ischemia in animal models of lung ischemia-reperfusion injury: is there a difference? Thorac Cardiovasc Surg. 2004;52(3):174-9.

17. King RC, Binns OA, Rodriguez F, Kanithanon RC, Daniel TM, Spotnitz WD, et al. Reperfusion injury significantly impacts clinical outcome after pulmonary transplantation. Ann Thorac Surg. 2000;69: 1681-5.

18. Ingemansson R, Massa G, Pandita RK, Sjoberg T, Steen S. Perfadex is superior to Euro-Collins solution regarding 24-hour preservation of vascular function. Ann Thorac Surg. 1995:60:1210-4.

19. Struber M, Hohlfeld JM, Fraund S, Kim P, Warnecke G, Haverich A. Low-potassium dextran solution ameliorates reperfusion injury of the lung and protects surfactant function. J Thorac Cardiovasc Surg. 2000:120:566-72.

20. Keshavjee SH, Yamazaki F, Yokomise H, Cardoso PF, Mullen JB, Slutsky AS, et al. The role of dextran 40 and potassium in extended hypothermic lung preservation for transplantation. $J$ Thorac Cardiovasc Surg. 1992;103(2):314-25.

21. Fischer S, Struber M, Haverich A. Current status of lung transplantation: patients, indications, techniques and outcome. Med Klin. 2002: 97:137-43.

22. Steen S, Kimblad PO, Sjoberg T, Lindberg L, Ingemansson R, Massa G. Safe lung preservation for twenty-four hours with Perfadex. Ann Thorac Surg. 1994;57:450-7.

23. Kimblad PO, Massa G, Sjoberg T, Steen S. Endothelium-dependent relaxation in pulmonary arteries after lung preservation and transplantation. Ann Thorac Surg. 1993;56:1329-33.

24. Abdalla EK, Caty MG, Guice KS, Hinshaw DB, Oldham KT. Arterial levels of oxidized glutathione (GSSG) reflect oxidant stress in vivo. J Surg Res. 1990:48:291-6.

25. Fischer S, Gohrbandt B, Struckmeier P, Niedermeyer J, Simon A, Hagl $\mathrm{C}$, et al. Lung transplantation with lungs from donors fifty years of age and older. J Thorac Cardiovasc Surg. 2005;129(4):919-25.

\section{Authoritative}

The Journal of Thoracic and Cardiovascular Surgery is the most frequently cited thoracic/cardiovascular surgery journal in the Science Citation Index. An article in JTCVS is cited on average almost twice as often as those in the closest cardiothoracic journal. 